\title{
Intraosseous Anomalous Drainage in a Patient with Varicose Veins: A Rare Anatomical Variant
}

\author{
Abdulrahman Alharbi ${ }^{1}$ (1) Yousof Al Zahrani ${ }^{2}$ Shaima Ahmed Abdulrahman ${ }^{2} \quad$ Mohammed Alsalman $^{1}$ \\ Mohammed Almoaiqel ${ }^{2}$
}

${ }^{1}$ Department of Medical Imaging, King Abdulaziz Medical City, Ministry of National Guard-Health Affairs, Riyadh, Saudi Arabia

2 Vascular and Interventional Radiology Unit, Department of Medical Imaging, King Abdulaziz Medical City \& King Abdullah Specialized Children's Hospital, Ministry of National Guard-Health Affairs, Riyadh, Saudi Arabia

Arab J Intervent Radiol 2021;5:116-118.

Intraosseous anomalous venous drainage of varicose veins is a rare anomaly. This is a 38-year-old lady who presented with left lower limb pain for 1 week. On inspection, there was erythema and mild swelling over anteromedial aspect of left lower extremity. On palpation of anterior aspect of lower third of left tibia, there was a firm mobile mildly tender mass.

X-ray of left lower extremity showed a small cortical defect at the anteromedial aspect of mid left tibia (-Fig. 1). This raised a suspicion for a possible osteoid osteoma. Computed tomography (CT) scan revealed a tubular structure crossing through the cortex of anterior midshaft of left tibia with intramedullary continuation superiorly and exiting through a defect at posterior superior aspect (-Fig. 2). It appeared to be connected to subcutaneous varicose veins. Magnetic resonance imaging (MRI) showed diffuse subcutaneous varicose veins with prominent pretibial varices that are directly connected to the anomalous intraosseous left tibia vein ( - Fig. 3 ). Further evaluation with left lower limb venogram ( - Figs. 4 and $\mathbf{5}$ ) demonstrated a network of mildly dilated veins with stagnation of the blood and prominent superficial veins at the level of the tibia.

Intra-osseous venous drainage of varicose veins in the lower extremities is rare. Most of the reported cases were unilateral involving the tibia.

published online January 19, 2022
Address for correspondence Abdulrahman Alharbi, MBBS, King Abdulaziz Medical City, Ar Rimayah, Riyadh 14611, Saudi Arabia (e-mail: alshidadi@gmail.com).
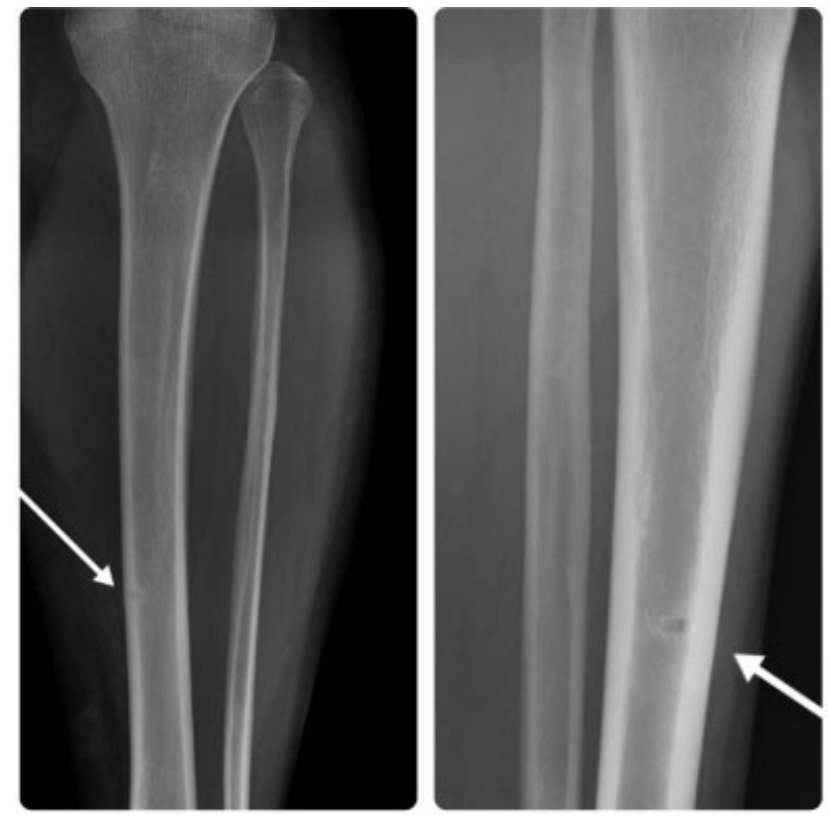

Fig. 1 Anteroposterior and lateral left tibia/fibula views demonstrate well-defined lucency (arrows) at the anteromedial aspect of mid tibia cortex. (c) 2022. The Pan Arab Interventional Radiology Society. All rights reserved.

This is an open access article published by Thieme under the terms of the Creative Commons Attribution-NonDerivative-NonCommercial-License, permitting copying and reproduction so long as the original work is given appropriate credit. Contents may not be used for commercial purposes, or adapted, remixed, transformed or built upon. (https://creativecommons.org/ licenses/by-nc-nd/4.0/)

Thieme Medical and Scientific Publishers Pvt. Ltd., A-12, 2nd Floor, Sector 2, Noida-201301 UP, India 


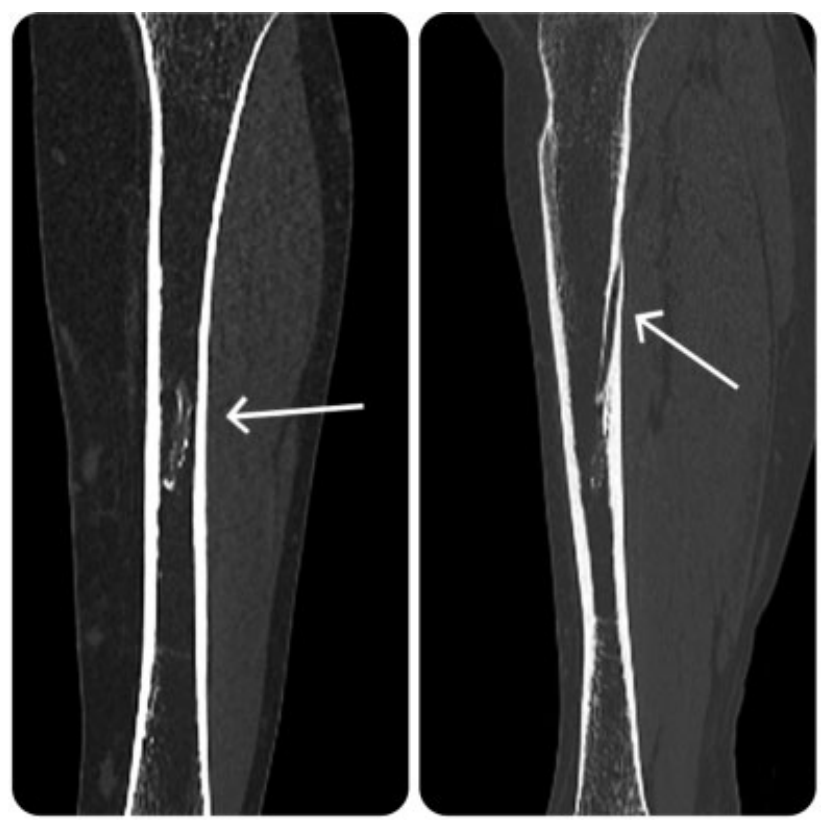

Fig. 2 Axial, coronal, and sagittal computed tomography cuts showing the intraosseous vein (arrows) coursing from anterior tibial cortex with intramedullary continuation and eventually exiting through the posterior cortex superiorly.
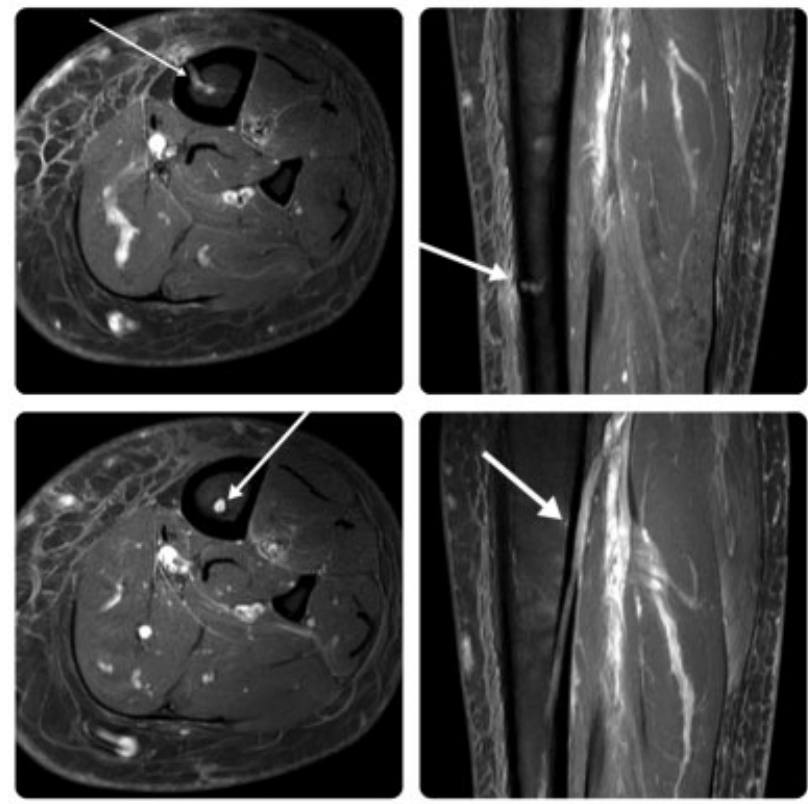

Fig. 3 Axial and sagittal magnetic resonance imaging cuts demonstrate the anomalous vein (arrows). There are multiple varicose veins in the surrounding soft tissue associated with subcutaneous edema.

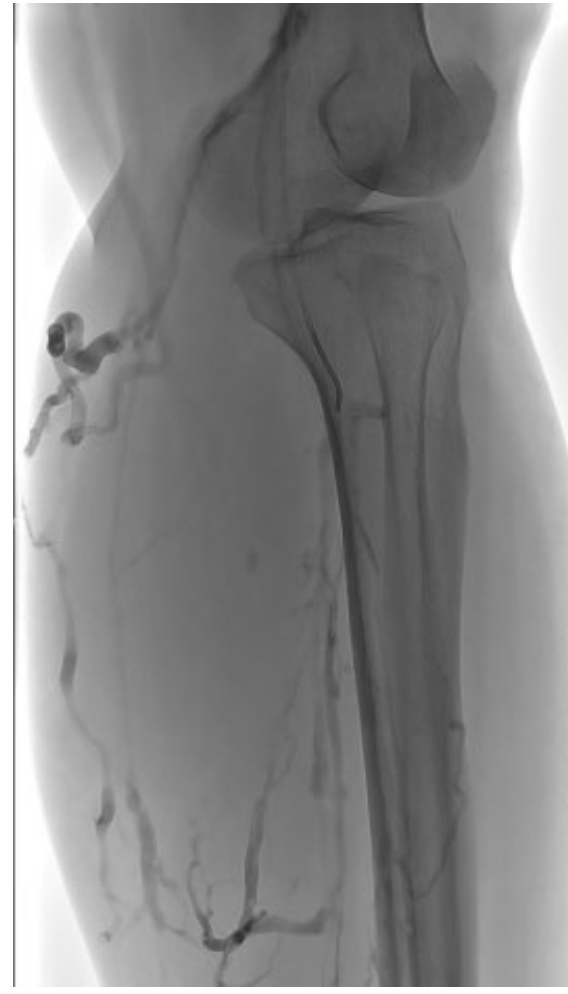

Fig. 4 Left lower limb venogram anterograde approach at the level of mid to distal third of the tibia demonstrates the network of mildly dilated veins and prominent superficial veins.

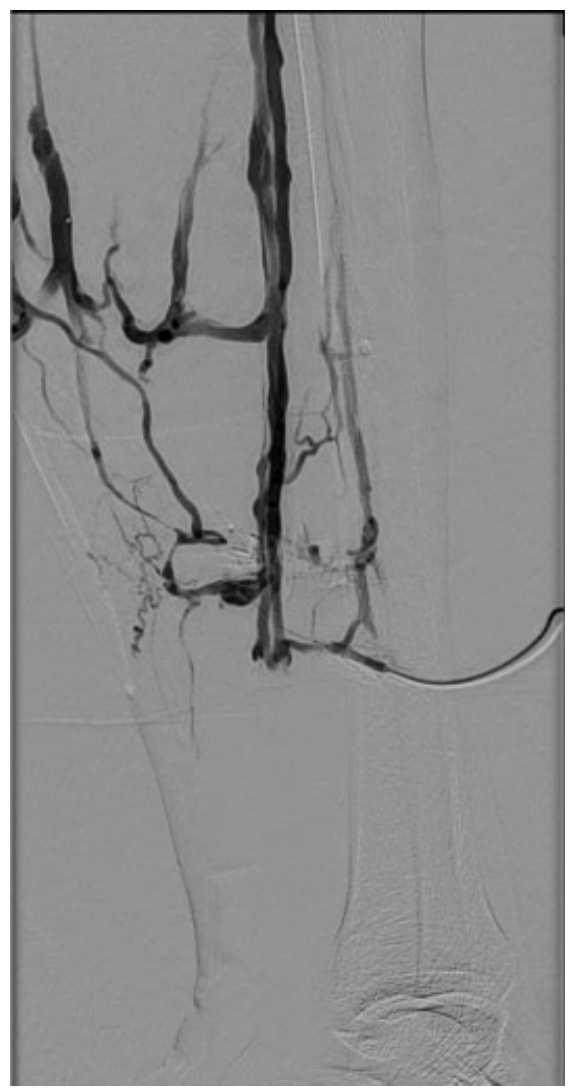

Fig. 5 Left lower limb venogram anterograde approach at the level of mid to distal third of the tibia demonstrates the network of mildly dilated veins and prominent superficial veins. 
118 Intraosseous Anomalous Drainage in a Patient with Varicose Veins Alharbi et al.

Intraosseous varicose veins can be diagnosed with color Doppler ultrasonography demonstrating reflux. However, cross-sectional imaging is important to differentiate varicose veins from other differential diagnoses such as arteriovenous malformation, arteriovenous fistula, or hemangioma. CT scan and MRI usually show a dilated intraosseous vein with direct connection to surrounding varicose veins. However, MRI is preferred due to better soft tissue resolution and absence of radiation.

Conflict of Interest

None. 